\title{
From 3D layout to dynamic simulation model
}

\author{
Alin Gavriluță $\breve{1}^{1, *}$, Ana Gavriluțăă ${ }^{1}$ Eduard-Laurențiu Nițu ${ }^{1}$, and Jérémie Schutz ${ }^{2}$ \\ ${ }^{1}$ University of Pitești, Department of Manufacturing and Industrial Management, Pitești, Romania \\ ${ }^{2}$ Université de Lorraine, LGIPM, EA 3096, Metz, F-57045, France
}

\begin{abstract}
The design of a supply system for an assembly line possess many challenges. A tool that helps the decision making and the adjustment of the supply system parameters is the dynamic simulation. This article has the aim of showing on a given case, the road from workstation layout, to its simulation model and an example of parameter that can vary and its effect of workstation performance. Starting from the 3D model of the workstation, varying the number of diversities present in the workstation, by using the simulation we can see the effect of this variation on the workstation cycle time.
\end{abstract}

\section{Introduction}

The globalization and strong competition in currentmarketplaces have forced companies to change theirways of doing business [1]. Presently, high productivity and a quick response to customers are essential for most producers [2]. Reducing the cycle time and inventories are objectives in managing production systems.

To enhance the system performance when designing layouts for assembly line workstation, the positions of the manufacturing system components must be appropriately determined by considering the sequence of tasks the operator conduct during the assembly process.

The purpose of this research is to present a methodology of crossing from layout to dynamic simulation for reducing the cycle time. To describe the methodology we used IDEF 0. Functional analysis methodology used in the study - IDEF0 [3] is based on abstraction and consists of the decomposition of the line activities in as fine details, until reaching the level of detail wanted.

\section{Methodology of crossing from layout to dynamic simulation}

To describe the working methodology of crossing from layout design to dynamic simulation and its functional links, it was used the IDEF0, functional modelling method.

Specific of this modelling method is the levelling of description from a higher point of view (A0) to as much details as needed. We consider the layout design and the simulation study as part of the overall design of the workstation and will be presented as so.

The workstation design has the following elements: input - process and environmental data; resources - software, tools of design and analysis, specialist, "know how"; controls -

${ }^{*}$ Corresponding author: gavrilutaalin@yahoo.com 
rules, norms and standards and as output - workstation design and operating mode, Figure 1.

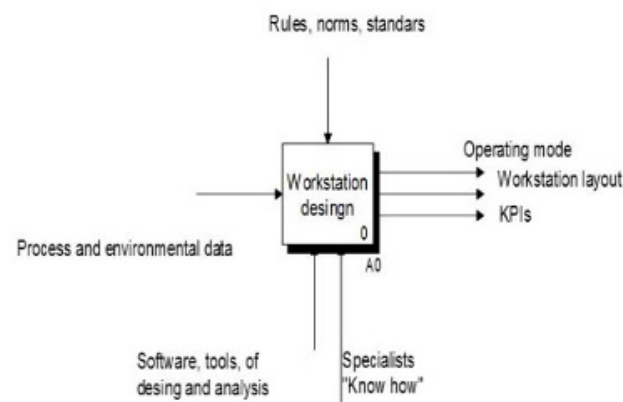

Fig.1.Workstation design model.

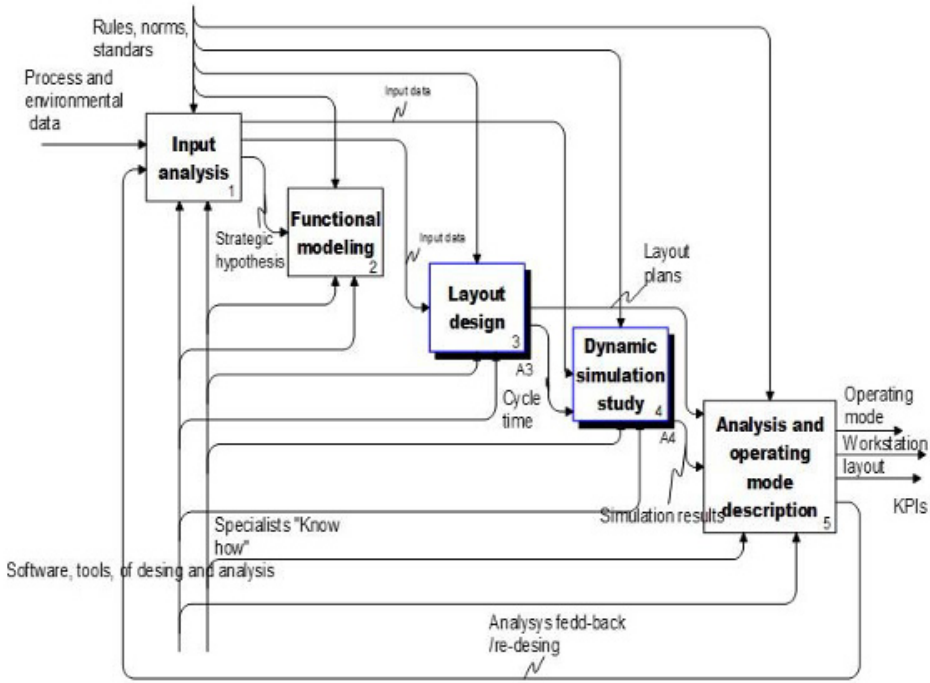

Fig.2.The steps of workstation design.

The first level of decomposition, Figure 2, has as elements:

-A1 - Input analysis: the entry data are processed and structured as to comply with the needs of design. Results are: strategic hypothesis that will guide the design steps and input data (cycle time, variables etc.) that will be the base of layout design and simulation;

- A2 - Functional modelling: as in this example, it helps in choosing elements as supply method, operation mode guidelines and explains the links between the system elements. The results are needed for layout design and dynamic simulation model construction;

- A3 - Layout design: uses the entry data and functional modelling guidelines to construct a layout and to determine the cycle time characteristics of it. Its results are used in dynamic simulation study and in the analysis step;

- A4 - Dynamic simulation study: starting from the functional model, this interactions are transformed in a dynamic model that will mimic the behaviour of a real life system, estimating the impact of strategic hypothesis, layout and variables from the design phase.

- A5 - Analysis and operating mode description: compiling the results of previous steps, the designers can compare performance KPIs concluding in the need of redesign or 
validation of results. As end products the designer delivers: workstations layouts, operating modes, KPIs (key performance indicator) estimations etc.

Giving a closer look at layout design, Figure 3, has as elements:

- A31 - Design of dependent elements: this elements are dependent of the supply method (conveyer, dynamic racks, trolleys etc.), of the method of workstation loading/unloading (manual, automatic, semiautomatic), of transportation of parts between workstations (on trolleys, conveyers, hangers etc.), or other dependency factors. The result of this step are elements as: support for components, dynamic racks, cranes, transport platforms;

- A32 - design of independent elements: this elements are given by process characteristics and are the same no matter what layout characteristic or supply method are used. The results are: tool stands, HMI (human machine interface) design, machining or assembly automatic workstations etc.

- A33 - workstation layout construction: taking all the designed elements, the layout designer builds the workstation variants and comparing the specific KPIs, choses the more performant ones.

- A34 - layout plan construction: this step consists in the construction of the system graphic design. Taking all workstations design, the designer places them in interdependency and ensures the correct flows. The results are layout plans and distance measurements between the elements of the layout. The distances and the positioning of elements in the $3 \mathrm{D}$ model is the base of time measurement and ergonomic conclusions to evaluate the layouts.

- A35 - distances and cycle time impact estimation: using the layout plan results the designer can make time measurements, ergonomic studies and variable estimations. This will be used in the dynamic simulation study.

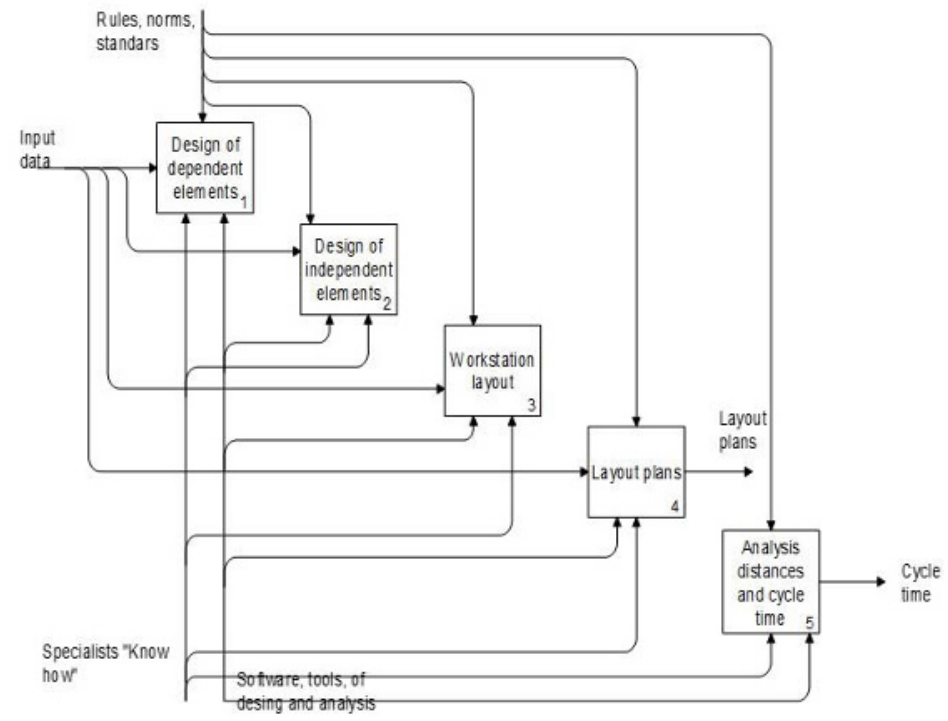

Fig.3.The steps of layout design.

This functional modelling can be used as base for further system design description upward and inward, depending on the needs. 


\section{Application of methodology on a given case}

\subsection{Workstation and operating mode description}

The presented example is an automotive assembly line workstation [4], Figure4. The work process is mainly manual and it contains dependent and independent elements. The assembly is made on a transport pallet that travels between workstations on a roller conveyer.
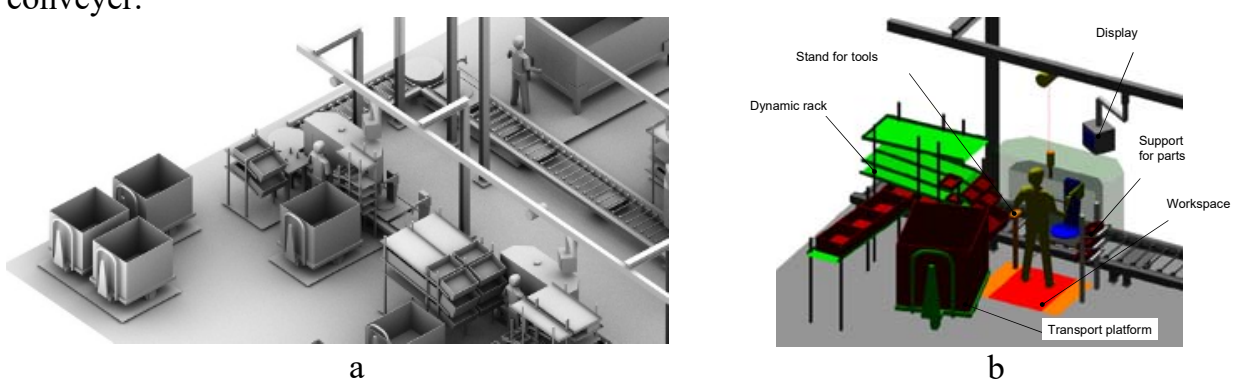

Fig.4. Workstation 3D model.

The core of the workstation is the strike point area. This is an area where the operator has the minimum effort and the maximum speed in movement. The aim of the designer is to keep the operator at maximum in this area in order to reduce the Non added Value due to operator movements.

The workstation assembles 4 types of products. At each cycle there are 9 parts (small, medium and large) assembled in a specific sequence. Of each part there have to be 2 hour of autonomy, this giving the capacity of storage on place (racks for small and medium parts, transport platform for large parts). The sequence of operation is concentrated in strike zone, exception being the movement to get the parts.
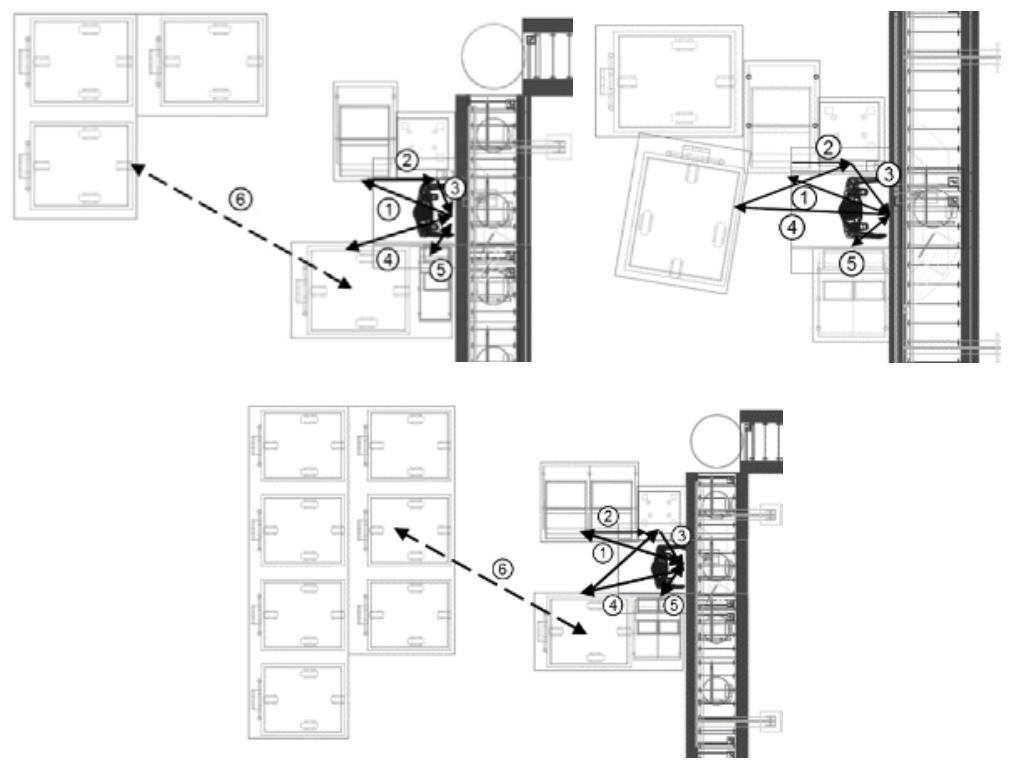

Fig.5. Sequence of operations impact in movement (1,2 and 3 diversities of parts). 
At each setup (batch change), the operator has to change the platform for large parts with the other type that is in the vicinity.

\subsection{Cycle time impact}

The cycle time needed to the assembly process is $1.2 \mathrm{~min}$. This we consider constant and independent of the layout design.

As example, the details of the time impact of the layout for 2 diversities of parts are presented in Table 1 and Table 2. The operations that appear with a specific frequency are very important, them giving the perturbation that influence the workstations efficiency and have an impact in the rest of the line (upstream and downstream).

Table 1.Sequence of operations impact in operator movements (2 diversities of parts).

\begin{tabular}{|c|c|c|c|c|}
\hline Sequence no. & Distance & No. of tours & No. steps & Time (min.) \\
\hline 1 & 1.5 & 1 & 3 & 0.048 \\
\hline 2 & 1.4 & 1 & 2 & 0.032 \\
\hline 3 & 0.6 & 1 & 1 & 0.016 \\
\hline 4 & 1.2 & 2 & 2 & 0.064 \\
\hline 5 & 0.5 & 4 & 1 & 0.064 \\
\hline 6 (setup) & 3.7 & 2 & 6 & 0.192 \\
\hline Total & 5.2 & - & 15 & $\mathbf{0 . 2 2 4}$ \\
\hline
\end{tabular}

Table 2.Packaging specificity and the impact of it on cycle time.

\begin{tabular}{|c|c|c|c|c|c|}
\hline Parts & Coef. & $\begin{array}{c}\text { No. } \\
\text { parts/ } \\
\text { pack. }\end{array}$ & $\begin{array}{c}\text { No. of } \\
\text { packs }\end{array}$ & $\begin{array}{c}\text { Freq. of } \\
\text { change } \\
\text { (hours) }\end{array}$ & Time (min.) \\
\hline R1 & 2 & 2900 & 1 & 29 & 0.053 \\
\hline R2 & 1 & 30 & 4 & 2.4 & 0.053 \\
\hline R3 & 1 & 100 & 1 & 2 & 0.053 \\
\hline R4 & 1 & 80 & 2 & 3.2 & 0.053 \\
\hline R5 & 6 & 1500 & 1 & 5 & 0.053 \\
\hline R6 & 1 & 1020 & 1 & 20.4 & 0.053 \\
\hline R7 & 1 & 250 & 1 & 5 & 0.192 \\
\hline R8 & 1 & 1500 & 1 & 30 & 0.053 \\
\hline R9 & 6 & 1500 & 1 & 5 & 0.053 \\
\hline
\end{tabular}

It can be observed that we have movements that are made at each cycle time and ones that are done depending on the supply method (autonomy, no. of parts in the packaging, setup at batch change), Figure5.

If it is to transpose this situation in a dynamic simulation model, it will be as following: - process cycle time - invariable or has small variations that can be with no impact. This is given by tools, machines, control equipment, etc.

- movement cycle time - this is specific and dependent of the layout characteristics. It can be input in simulation as a variable cause of the high variations due to operator influence. This will also vary from one diversity to another due to different distance implied;

- change of packaging cycle time - this is specific and dependent of the supply method and the diversity number, that gives the distances in the workplace. It can be input in simulation with a cyclic currency (ex. at each 250 work cycles, the capacity of packaging for the component R7, at 2 diversities variant, the operator has a 0,192 min. packaging change time);

- batch change cycle time due to process - the time needed for tools change, machines setup, etc. This cycle is independent of the layout; 
- batch change cycle time due to layout: considering the method of supply and the position of the alternative parts that have to be assembled, this cycle time can be with great influence on the line flexibility. For example: on an assembly line were the process setup is " 0 ", the supply setup is the only responsible of line flexibility at batch change.

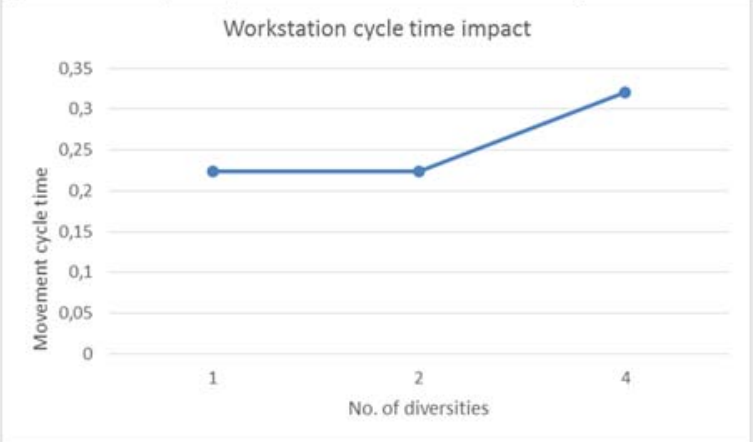

Fig.6. Results of analysis of movement on the workstation cycle time.

\section{Conclusions}

The layout design has a strong impact on the dynamic simulation analysis. The tendency can be to take into account the direct process time and setup. As started to show in this article, the layout has an import influence, if not more of an importance than the breakdown.

In our example the cycle time direct linked with the process is $1.2 \mathrm{~min}$. Making the sum of all movement done per cycle the cycle time ends to be from 1.42 to $1.54 \mathrm{~min}$, concluding that the impact of the layout is between 15 to $22 \%$ from the workstation cycle time (fig 6 ).

Also it can be viewed that in a line where the setup at batch change can be 0 , which theoretically can be completely flexible, due to logistic reconfiguration of workstation, leads to a decrease of $13 \%$ the line productivity.

The production line system, and more specific the assembly line system is more and more a concept of batch of 1, reduction of WIP (work in process) (small if no buffers between workstations), inline conveyer transport. These concepts imply that any cycle time variation has a strong influence in the overall system. To detect this influence, it is discovered the need of dynamic simulation of flow, and in it the analysis of the impact of workstation layout.

The next step of the study is to extend the analysis from one workstation to a full assembly line.

\section{References}

1. R.S. Chena, K.Y.Lua, S.C.Yua, H.W.Tzenga, C.C. Chang, Information and Management 41 25-37 (2003)

2. J.D. Wells, W.L. Fuerst, J. Choobineh, Information and Management 3553-62(1999)

3. A. Rotaru, A. Gavriluţă, E. Niţu, Applied Mechanics and Materials 371 116-120(2013)

4. A. Gavrilută, E. Niţu, A. Rotaru, Proceedings of the 3th Review of Management and Economic Engineering - International Management Conference "A new dilemma: between East and West" 263-274 (2012) 